\title{
Bevacizumab als dritte Substanz beim fortgeschrittenen NSCLC?
}

\author{
Der VEGF-Antikörper ist etablierter Bestandteil in der Therapie \\ mehrerer solider Tumore. In einer Metaanalyse prüfte ein Team den \\ Effekt von Bevacizumab zusätzlich zu einer platinbasierten Doublet- \\ Chemotherapie in der Erstlinientherapie des nichtkleinzelligen \\ Lungenkarzinoms (NSCLC).
}

\begin{abstract}
$\mathrm{n}$ ihre Auswertung nahmen die Forscher um Jean-Charles Soria zwei Phase-II-Studien (JO19907, AVF-0757g) sowie die Phase-III-Studien ECOG 4599 und AVAiL auf. In AVAiL erhielten die Studienteilnehmer als Erstlinientherapie Cisplatin und Gemcitabin, in den anderen drei Studien Carboplatin und Paclitaxel. Per Randomisierung erfolgte die Chemotherapie mit oder ohne zusätzliche Gabe von Bevacizumab. Der VEGFInhibitor wurde in allen Studien in dreiwöchigen Abständen für bis zu sechs Zyklen gegeben. Die Dosierung lag bei 15 $\mathrm{mg} / \mathrm{kg}$ Körpergewicht in ECOG 4599 und JO19907; in den beiden anderen Studien gab es zwei Dosierungen mit 7,5 mg oder $15 \mathrm{mg} / \mathrm{kg}$. Zur Auswertung kamen
\end{abstract}

Daten von 2.194 Patienten mit den Tumorstadien IIIB und IV, 1.313 von ihnen erhielten Bevacizumab.

Beim Gesamtüberleben gab es ein signifikantes Ergebnis zugunsten der Bevacizumab-Addition (Hazard Ratio [HR] 0,90; $p=0,03)$. Die Wahrscheinlichkeit ein Jahr zu überleben war unter dem VEGF-Antikörper um $4 \%$ höher (55 vs. $51 \%$ der Patienten). Auch das progressionsfreie Überleben fiel unter Bevacizumab günstiger aus (HR 0,72; $\mathrm{p}<0,001$ ).

Faktoren, die den Effekt von Bevacizumab signifikant verstärkten, waren das Vorliegen von Adenokarzinomen sowie eine Abnahme des Body-Mass-Index der Patienten von maximal 5\%. Bevacizumab erhöhte jedoch das Risiko einer
Grad-3- oder Grad-4-Nebenwirkung für Proteinurie (um den Faktor 4,8), Hypertonie $(3,7)$, Hämorrhagie $(2,7)$, febrile Neutropenie $(1,7)$ und Neutropenie $(1,5)$.

Fazit: Gesamtüberleben und progressionsfreies Überleben waren in dieser Analyse durch die Bevacizumab-Gabe verlängert. In einem Kommentar widersprechen Everett Vokes und Kollegen dieser Interpretation. Ihrer Meinung nach trägt in erster Linie der positive Effekt aus der ECOG-Studie zum günstigen Ergebnis der Analyse bei, in die zudem keine individuellen Patientendaten, sondern nur gepoolte Gesamtstudiendaten eingingen.

Andreas Fischer

Soria J-C et al. Systematic review and metaanalysis of randomised, phase II/III trials adding bevacizumab to platinum-based chemotherapy as first-line treatment in patients with advanced non-small-cell lung cancer. Ann Oncol. 2013; 24(1):20-30. - Kommentar: Vokes E et al. Evidence-based role of bevacizumab in non-small cell lung cancer. ibidem 6-9.

\section{NSCLC: Biomarkersuche mit Überraschung}

Die Ribonukleotidreduktase-Untereinheit M (RRM1) wurde bisher für einen möglichen Biomarker für das Ansprechen eines nichtkleinzelligen Lungenkarzinoms (NSCLC) auf Gemcitabin im Rahmen einer platinbasierten Chemotherapie gehalten. Eine Studie konnte das nicht bestätigen, fand aber einen anderen Zusammenhang.

\begin{abstract}
RM1 ist ein wichtiger regulierender Faktor der Ribonukleosid-Diphosphat-Reduktase, eines Schlüsselenzyms der DNA-Synthese. Der Antimetabolit Gemcitabin setzt dessen Funktion herab, indem er den Pool an DNA-Bausteinen verringert. Nachdem einige Studien positive Hinweise auf eine mögliche Rolle von RRM1 als Biomarker für die Gemcitabin-Sensitivität gezeigt hatten, prüften dänische Autoren dies retrospektiv anhand der Gewebeproben von $261 \mathrm{~Pa}$ tienten mit NSCLC aus einer Phase-IIIStudie, in der bei insgesamt 443 Lungenkrebspatienten die Kombination Paclitaxel, Cisplatin und Gemcitabin mit Cisplatin plus Vinorelbin verglichen worden war.
\end{abstract}

Die Untersuchungen zeigten keine Korrelation zwischen dem immunhistochemisch nachgewiesenen RRM1 und der Krankheitskontrolle, dem progressionsfreien Überleben (PFS) und dem Gesamtüberleben (OS) bei den Patienten des Gemcitabin-Arms.

Zur Überraschung der Untersucher könnte RRM1 aber als Biomarker für die Sensitivität auf Vinorelbin infrage kommen, obwohl man bisher davon ausgegangen war, dass diese Substanz als Spindelgift über einen anderen zytostatischen Mechanismus wirkt als Gemcitabin: Die Tumorkontrollrate lag bei RRM1-negativen Tumoren im Vinorelbin-Arm mit 68,8\% deutlich über dem der Patienten mit RRM1-positivem Tu- mor $(31,2 \%, p=0,046)$. Das PFS lag bei 6,96 bzw. 3,93 Monaten ( $\mathrm{p}=0,000$ ), das mediane OS bei 11,57 versus 7,4 Monaten $(\mathrm{p}=0,002)$.

Die multivariate sowie die Interaktionsanalyse ergaben als einzigen weiteren klaren prädiktiven Marker einen negativen RRM1-Status bei gleichzeitigem Vorliegen eines Adenokarzinoms mit einer Hazard Ratio für Tod von deutlichen $0,36(\mathrm{p}=0,000)$.

Fazit: Die RRM1-Expression scheint bei der Kombinationstherapie mit Cisplatin, Paclitaxel und Gemcitabin keine Prädiktion des Therapieerfolgs zuzulassen. Dagegen ergab sich eine überraschend deutliche Aussagekraft des RRM1-Status für einen Therapieerfolg im Kontrollarm. Zu klären ist nun, so die Autoren, welche Rolle RRM1 für die Sensitivität gegenüber Vinorelbin spielt.

Friederike Klein

Vilmar AC et al. Predictive impact of RRM1 protein expression on vinorelbine efficacy in NSCLC patients randomly assigned in a chemotherapy phase III trial. Ann Oncol. 2013;24(2):309-14. 